Revue

Revue de l'histoire des religions

del'histoire des religions

Un dieu peut-il mourir ? Enquête sur la fin des cultes dans l'Antiquité gréco-romaine

\title{
Les discours chrétiens sur la «fin des cultes » publics au Levant : l'argument des sanctuaires
}

Christian discourses on the "end of cults" in the Levant: the argument of cult places

Nicole Belayche

\section{(2) OpenEdition}

Journals

Édition électronique

URL : http://journals.openedition.org/rhr/8901

DOI : 10.4000/rhr.8901

ISSN : 2105-2573

Éditeur

Armand Colin

Édition imprimée

Date de publication : 1 juin 2018

Pagination : 209-232

ISBN : 978-2-200-93177-3

ISSN : 0035-1423

Référence électronique

Nicole Belayche, "Les discours chrétiens sur la « fin des cultes » publics au Levant : I'argument des sanctuaires », Revue de l'histoire des religions [En ligne], 2 | 2018, mis en ligne le 01 juin 2020, consulté le 13 janvier 2021. URL : http://journals.openedition.org/rhr/8901 ; DOI : https://doi.org/10.4000/rhr. 8901 


\section{Les discours chrétiens sur la « fin des cultes » publics au Levant : l'argument des sanctuaires}

La «fin des cultes» est plus facile à repérer dans les sanctuaires, disparus par destruction volontaire, abandon ou réaffectation. Les religions traditionnelles, et leurs sociétés, sont construites sur la spatialité : l'espace sacré est à la fois le « lieu » du dieu et un lieu de repère culturel et identitaire qui dépasse le cadre rituel. Les discours chrétiens sur la "fin des cultes", parce qu'ils ont polémiqué avec des conceptions religieuses spatialisées ( "paganisme » et judaïsme), se sont emparés de cette symbolique spatiale pour écrire le récit de la christianisation au Levant - à confronter au modèle utopien de Jonathan Z. Smith - et dessiner des exempla de saints hommes. Avec trois cas du IV siècle (Aelia Capitolina, Aphaca/Héliopolis et Gaza), on analyse l'argument des sanctuaires dans ces discours.

\section{Christian discourses on the "end of cults" in the Levant: the argument of cult places}

The "end of cults" is easier to pinpoint in sanctuaries, which disappeared after either voluntary destruction, abandonment or reoccupation. Traditional religions, and their societies, are built on spatiality : the sacred place is both the "locus" of the divinity and a cultural and identity landmark that goes beyond its ritual use. Because Christian discourses on the "end of cults" were engaged with spatialized religious conceptions ("paganism" and Judaism), they took over this spatial symbolism for writing the story of Christianization in the Levant - thus challenging the utopian model of Jonathan Z. Smith - and for drawing the exempla of holy men. On the basis of three cases from the fourth century (Aelia Capitolina, Aphaca/Heliopolis, and Gaza), this paper analyzes how these discourses used the argument of sanctuaries. 
«La demeure des démons est devenue la maison de Dieu, la lumière du Seigneur a brillé là où les ténèbres la cachaient, là où se tenaient les sacrifices des idoles sont maintenant les chœurs des anges $»^{1}$.

Dans une lettre désormais bien connue qu'il écrivit à son maitre Hermann Usener depuis Constantinople le 11 mars 1900, Franz Cumont confiait « [s]a résolution [...] d'écrire un jour [...] une histoire de [1]a disparition [du paganisme] - si vraiment il a disparu $»^{2}$. Joseph Geiger ne parle pas autrement près de cent ans plus tard : « a history, properly so called, of the end of paganism cannot perhaps ever be written $»^{3}$. Ce sont deux expressions parmi d'autres de la difficulté à fixer sur une échelle chronologique la «fin des cultes » pour employer une expression plus juste - puisque religio, id est cultus deum selon la formule de Cicéron. D'une part, comme le rappelle opportunément Christopher P. Jones, le «paganism was indestructible, in that it had never really existed except as en entity in the eyes of its opponents $»^{4}$, d'autre part la chronologie diffère selon qu'on examine la disparition des sanctuaires (par destruction volontaire, abandon ou réaffectation ${ }^{5}$ ) ou des pratiques rituelles.

1. Orientis Graeci Inscriptiones Selectae, éd. Wilhelm Dittenberger (dorénavant : $O G I S$ ), II, 610, 1. 1-3, en 515-516, au-dessus de la porte de l'église : $\Theta \varepsilon o \tilde{v}$

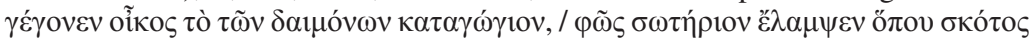

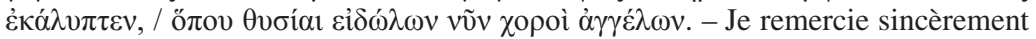
Karine Mackowiak et Christian Stein pour leur invitation à participer à cette réflexion, qui me permet de revenir sur des faits que j'avais abordés jusqu'alors de façon dispersée.

2. Corinne Bonnet, Le " grand atelier de la science ». Franz Cumont et l'Altertumswissenschaft. Héritages et émancipations, t. 2, Bruxelles, Rome, Institut Historique Belge de Rome, 2005, p. 200, nº 20 (je souligne).

3. John Geiger, « Aspects of Palestinian Paganism in Late Antiquity », Sharing the Sacred. Religious Contacts and Conflicts in the Holy Land, éd. Arieh Kofsky, Guy G. Stroumsa, Jérusalem, Yad Izhak Ben Zvi, 1998, p. 3.

4. Christopher P. Jones, Between Pagan and Christian, Harvard, University Press, 2014, p. 145.

5. Sur la difficulté de déterminer les raisons à partir de l'archéologie: Bryan Ward-Perkins, «The End of the Temples : An Archaeological Problem », Spätantike Staat und religiöser Konflikt. Imperiale und locale Verwaltung und die Gewalt gegen Heiligtümer, éd. Johannes Hahn, Berlin, New York, De Gruyter, 2011, p. 187-199. Pour une combinaison des « perspectives of monument, event and discourse »: From Temple to Church. Destruction and Renewal of Local 
L'enquête fait réfléchir sur la pertinence heuristique des modèles capables de rendre compte des mutations religieuses: parmi ceux-ci, la taxinomie spatiale établie par Jonathan Z. Smith entre le « locatif » (un espace clos orienté vers un centre, le temple, dans lequel rôles et statuts sont déterminés) et l' «utopien » (un espace ouvert, fluide et centripète, aux frontières poreuses, dont la carte - i.e. le groupe religieux - n'est ni dans ni sur le territoire) $)^{6}$, qui sert aujourd'hui de cadre théorique à toute une recherche sociologisante anglo-saxonne, principalement américaine.

\section{LA SPATIALITÉ DES RELIGIONS RITUALISTES}

Pour les religions ritualistes antiques, l'immanence d'un dieu dans le monde des hommes dépendait des hommages cultuels qu'il recevait sur son autel dans son lieu, puisque le culte est un système de communication entre dieux et hommes ${ }^{7}$. L'agencement de ce lieu répondait à l'identité de l'interlocuteur - dans son ontologie (en tant que puissance surnaturelle) comme dans ses champs et modalités de compétence ${ }^{8}$. Tout athanatos/immortalis qu'il fut,

Cultic Topography in Late Antiquity, éd. Johannes Hahn, Stephen Emmel, Ulrich Gotter, Leyde, Boston, Brill, 2008, p. 6. Pour un panorama factuel, voir Luke Lavan, « The End of the Temples : Towards a New Narrative », The Archaeology of Late Antique "Paganism", éd. L. Lavan, Michael Mulryan, Leyde, Boston, Brill, 2011, p. XIX-XLIX.

6. Jonathan Z. Smith, « Map is not the territory », in J. Z. Smith, Map is not the territory. Studies in the History of Religions, Leyde, Brill (« Studies in Judaism in Late Antiquity », 23), 1978, p. 289-309; Magie de la comparaison et autres études d'histoire des religions, éd. Daniel Barbu, Nicolas Meylan, Genève, Labor et Fides, 2014, p. 147-173. Voir aussi «The Topography of the Sacred», in J. Z. Smith, Relating Religion. Essays in the Study of Religion, Chicago, University of Chicago Press, 2004, p. 101-116.

7. Voir Philippe Borgeaud, «Réflexions sur l'histoire de la comparaison en histoire des religions antiques», Mètis, t. 1 (n.s.), 2003, p. 9-33, ici p. $20:$ «le respect scrupuleux non pas d'un objet ultime (la divinité), mais de l'instrument de la médiation. Ce qu'il faut respecter, ce sont les modalités traditionnelles de la communication avec le surhumain, ou l'invisible». Voir aussi Jörg Rüpke, «Antike Religionen als Kommunicationssystem», Gebet und Fluch, Zeichen und Traum. Aspekte religiöser Kommunication in der Antike, éd. Kai Brodersen, Münster, Lit (« Antike Kultur und Geschichte», 1), 2001, p. 13-30.

8. Voir Puissances divines à l'épreuve du comparatisme: constructions, variations et réseaux relationnels, éd. Corinne Bonnet, Nicole Belayche, Marlène Albert LLorca et al., Turnhout, Brepols (Bibliothèque de l'École des hautes études. Sciences religieuses, 175), 2017. 
un dieu pouvait « mourir », c'est-à-dire disparaître, s'il ne recevait plus de culte. Le satiriste du $\mathrm{II}^{\mathrm{e}}$ siècle Lucien de Samosate, fortement inspiré par une conception épicurienne, s'en est gaussé lorsqu'il met dans la bouche du rôle-titre de Zeus tragédien cette alternative existentielle induite par le culte: «si nous devons encore être honorés et recevoir les offrandes $(\tau \mu \mu \tilde{\alpha} \sigma \theta \alpha \imath \dot{\eta} \mu \tilde{\alpha} \varsigma$ हैं

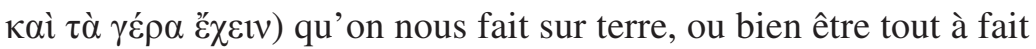

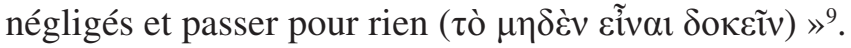

Artémis ne s'était-elle pas vengée d'Enée en envoyant le sanglier que tua son fils Méléagre, parce que le roi de Calydon ne l'avait pas invitée au sacrifice des Thalysies? «Les autres dieux se rassasiaient d'hécatombes ; à elle seule CEnée n'avait rien offert, à la fille du grand Zeus, qu'il l'eût oubliée ou n'y eût pas

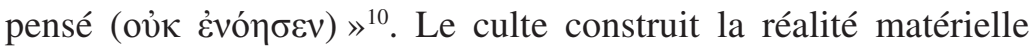
de la divinité dans un espace qui est le sien ${ }^{11}$. Les sanctuaires, espaces institutionnalisés de la rencontre entre une divinité et ses dévots, étaient réputés coexistants à l'organisation politique. Dans les villes, écrit Libanios à la fin du IV siècle, « les premiers

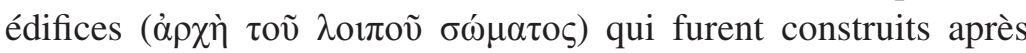

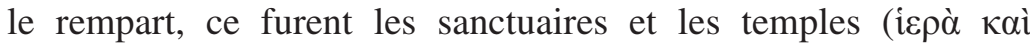
$v \varepsilon(\hat{)}) \gg^{12}$. Installer une divinité signifiait lui attribuer un espace propre avec un autel. À Rome, supprimer un dieu, le négliger (neg-legere), voire seulement le déplacer, ne pouvait se faire sans impiété puisque le temple (ou l'autel) est consacré par le culte $^{13}$ et qu'il est par conséquent la propriété du dieu. Déplacer une puissance divine réclamait un rituel d'exauguratio au cours duquel la divinité manifestait par des signes sa décision de quitter

9. Lucien, Zeus tragique 3 ; cf. aussi 18. Chez Platon, Symposion 190c-d, les dieux hésitent à détruire l'humanité par crainte de ne plus recevoir d'offrandes.

10. Voir Iliade, IX, 535-537; le verbe noein entre fréquemment dans l'expression de la conception des dieux.

11. Michael Lipka, Roman Gods. A conceptual approach, Leyde, Boston, Brill (Religions in the Graeco-Roman World, 167), 2009, a posé correctement l'espace comme l'un des principes constitutifs de la définition des dieux romains, mais surinterprété la rupture avec le christianisme. Voir infra.

12. Libanios, Pro templis (Oratio 30), 4, trad. R. Van Loy, Byzantion, t. 8, 1933, p. 21.

13. Voir Vinciane Pirenne Delforge, «Des marmites pour un méchant petit Hermès ! ou comment consacrer une statue », Image et religion dans l'Antiquité gréco-romaine, éd. Sylvia Estienne, Dominique Jaillard et alii, Naples, Centre Jean Bérard, École française d'Athènes, 2008, p. 103-110. 
ou non le lieu. Ainsi Terminus refusa-t-il, selon la tradition, de quitter sa résidence capitoline qu'avait choisie Tarquin le Superbe pour y installer Jupiter ${ }^{14}$. Toutefois, tant que son sanctuaire existe encore, ce n'est pas parce qu'une divinité ne semble plus recevoir d'honneurs qu'elle est «morte», comme le prouve le lucus des nymphes Furrinae sur le Janicule à Rome. Il ne semble plus accueillir les Furrinalia depuis l'époque républicaine, d'après les traces conservées. Or au $\mathrm{II}^{\mathrm{e}}$ siècle de notre ère, lorsque le sanctuaire a été loué à une communauté de Syriens qui y vénère son dieu, une dévote phénico-chypriote a rendu hommage à son Baal ancestral « interprété » en adjoignant la mention des Nymphes à la fin de la dédicace : «À Zeus Keraunios ( $\Delta$ ı̀ Kepavví $($ ), Artémis appelée aussi Sidonienne de Chypre, à la suite d'un ordre (du dieu) a

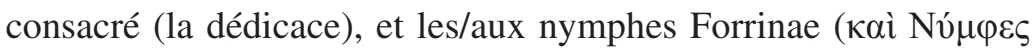
Форрі́ves) $»^{15}$.

La question de la «fin des cultes», surtout lorsqu'ils sont publics, croise donc nécessairement celle du devenir des sanctuaires. Eusèbe de Césarée, père d'un récit chrétien de l'histoire et hagiographe de Constantin, s'est servi de lieux de culte levantins pour nourrir son discours de l'avènement d'une nouvelle ère, alors qu'il n'y avait aucune «offensive » contre les lieux de culte païens... si tant est qu'elle ait jamais été lancée. Les enquêtes archéologiques récentes montrent que les destructions de temples furent peu nombreuses ${ }^{16}$ en regard du déploiement de l'« idéologie de la destruction $\gg^{17}$ qu'a véhiculée la littérature chrétienne.

14. Tite-Live, I, 55, 2-4 et V, 54, 5. Voir Fay Glinister, «Sacred rubbish », Religion in Archaic and Republican Rome and Italy : Evidence and Experience, éd. Edward Bisham, Christopher Smith, Édimbourg, Edinburgh University Press, 2000, p. 66-67.

15. Corpus Inscriptionum Latinarum (dorénavant: CIL), VI, $36802=$ OSUR, 1996 , p. $58, \mathrm{n}^{\circ} 1$. Voir aussi $C I L$, VI, $422=30765$ : « Consacré à Jupiter très bon très grand héliopolitain Auguste; au Génie des Forinae et aux fidèles de ce lieu (cultoribus huius loci)...».

16. Supra n. 4 pour le peu de support de l'archéologie à cette thèse. Pour la question spécifique des temples, voir Friedrich W. Deichmann, « Frühchristliche Kirchen in antiken Heiligtümern », Jahrbuch des Deutschen Archäologischen Instituts, t. 54, 1939, p. 105-136, et Richard P.C. Hanson, « The Transformation of Pagan Temples into Churches in the Early Christian Centuries », Journal of Semitic Studies, t. 23, 1978, p. 257-267.

17. David Frankfurter, «Iconoclasm and Christianization in Late Antique Egypt: Christian Treatments of Space and Image », From Temple to Church, p. $135-160$. 
Pour l'historien des religions, ce discours de «l'autre » contribue à valider le rôle cardinal de l'espace sacré lorsque les cultores deum construisaient leurs dieux comme puissances ${ }^{18}$, et des lieux de culte comme signaux visuels et lieux de repère culturels et identitaires, bien au-delà du cadre ritue ${ }^{19}$. Je propose de mettre en évidence comment les discours chrétiens se sont coulés dans cette symbolique spatiale «païenne» pour exprimer une exception chrétienne et dessiner des exempla de saints hommes, empereur ou évêque, parce que les cultes païens dont ils étaient les thuriféraires ${ }^{20}$ étaient eux-mêmes construits sur la spatialité. Les lieux de culte étaient des marqueurs identitaires communautaires que les empereurs ont protégés pour garantir la laetitia de leurs sujets $^{21}$; et ils sont demeurés un référent essentiel même pour une religion universaliste de salut. Cette observation oblige à utiliser sans dogmatisme la typologie spatiale de Jonathan $\mathrm{Z}$. Smith $^{22}$, dont il a affiné les contours en 2003 en proposant trois types religieux répondant à des «stratégies topographiques» (des spatialités) différentes : le here, le there et le anywhere ${ }^{23}$, c'est-à-dire le ici (la religion domestique), le là (« des religions civiques ou étatiques, centrées autour des temples»), et, surtout, le où-que-ce-soit - qui reconfigure et réinterprète des « éléments caractéristiques des religions de "l'ici" et du "là" ", accède «à une relative prééminence dans le contexte de l'Antiquité tardive et dans l'ensemble du monde méditerranéen » et rend

18. Voir Odile Journet-Diallo, « De la captation d'une instance à la fabrication d'une puissance. Les ukíin jóola (Sénégal/Guinée-Bissau)», Puissances divines, p. 363-377. Plus généralement Béatrice Caseau, «Sacred Landscapes », éd. Glen W. Bowersock, Peter Brown, Oleg Grabar, Late Antiquity: A Guide to the Postclassical World, Harvard, Harvard University Press, 1999, p. 21-57.

19. Tesse D. Stek, Cult Places and Cultural Change in Republican Italy: A Contextual Approach to Religious Aspects of Rural Society after the Roman Conquest, Amsterdam, Amsterdam University Press ( « Amsterdam Archaeological Studies », 14), 2009, et pour l'Antiquité tardive N. Belayche, « Des lieux pour le "profane" dans l'Empire tardo-antique ? Les fêtes entre koinônia sociale et espaces de rivalités religieuses », Antiquité tardive, t. 15, 2007, p. 35-46.

20. Pour un tableau des grandes tendances historiographiques, Béatrice Cazeau, «Christianisation et violence religieuse : le débat historiographique », Chrétiens persécuteurs. Destructions, exclusions, violences religieuses au IV siècle, éd. MarieFrançoise Baslez, Paris, Albin Michel, 2014, p. 11-36.

21. Voir N. Belayche, « Des lieux pour le "profane" », p. 38-39.

22. Supra n. 6.

23. Jonathan Z. Smith, Relating Religion, «Here, There, and Anywhere», p. 323-339 (= Magie de la comparaison, p. 81-101). 
compte selon lui des religions « de salut», donc de la révolution chrétienne ${ }^{24}$. Les discours chrétiens sur les sanctuaires levantins ici analysés résistent à cet «où-que-ce-soit» qui permettrait de penser l'événement fondateur pour l'Occident (le passage des religions ritualistes traditionnelles au christianisme), parce qu'ils ont polémiqué avec des conceptions religieuses fortement spatialisées, les religions traditionnelles et le judaïsme à Jérusalem.

En examinant la « fin des cultes » publics au Levant, en SyriaPhoenicia et Syria-Palaestina, on distinguera les témoignages sur les sanctuaires (le «there » de Jonathan Smith) des pratiques cultuelles en général, car, à la différence de lieux de culte identifiés, les pratiques sont plus labiles historiquement, comme en termes de documentation. Toutes les sources antiques en témoignent, quelle que soit leur affiliation religieuse, depuis un païen du $\mathrm{I}^{\text {er }}$ siècle $^{25}$ jusqu'à un historien ecclésiastique sur les dévots de Mambré près de Hébron au $\mathrm{V}^{\mathrm{e}}$ siècle $^{26}$, en passant par les témoignages rabbiniques sur les pratiquants de l'avoda zara (le culte étranger) au III $^{\mathrm{e}}$ siècle $^{27}$ et la loi d'interdiction des pratiques païennes en $392^{28}$. Ces pratiques éphémères ne laissent que peu de traces, un peu plus désormais grâce aux méthodes de l'archéologie du rituel. Pour le IV siècle choisi pour cette étude du fait de son statut charnière dans les mutations religieuses tardo-antiques, mises à part les rarissimes découvertes archéologiques, la documentation qui provient avant tout des récits d'auteurs chrétiens insiste particulièrement sur des sanctuaires. Au Proche-Orient, les situations furent variées du début

24. Ibid., Magie de la comparaison, p. 94 et 84.

25. Sénèque, De la superstition, ap. Augustin, Cité de Dieu, VI, 10 : « Monte au Capitole [...] celui-ci le nettoie, celui-là le parfume, imitant par le mouvement de son bras le geste du masseur. Voici des femmes qui coiffent Junon et Minerve... ».

26. Sozomène, Histoire ecclésiastique (dorénavant : HE), II, 4, 5 : « selon une

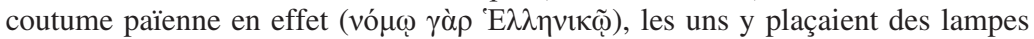
allumées, d'autres y [dans le puits] jetaient du vin ou des gâteaux, d'autres des pièces de monnaies ou du parfum ou de l'encens ».

27. M Sanhedrin, 7, 6: «Celui qui fait des offrandes, apporte de l'encens, verse des libations », etc. ; voir aussi 4, 2 et TJ Sanhedrin, 7, 6.

28. Code Théodosien (dorénavant : CTh), xvI, 10, 12 (8 novembre 392) : que personne «ne sacrifie à des statues privées de sentiment une victime innocente ou par une impiété plus discrète, ne vénère un lare par le feu, un génie par le vin nouveau, les pénates par le parfum, n'allume des lampes, n'offre de l'encens, ne suspende des guirlandes ». 
à la fin du $\mathrm{IV}^{\mathrm{e}}$ siècle $^{29}$, et les discours diffèrent derrière une ligne générale commune.

\section{LES SANCTUAIRES PAÏENS INDIQUÉS COMME DÉTRUITS EXPRIMENT UNE PROFESSION DE FOI}

Selon les auteurs ecclésiastiques, Constantin a fait détruire deux sanctuaires païens en Palestine : celui du Golgotha à Aelia Capitolina et celui de Mambré près de Hébron ${ }^{30}$. En SyriePhénicie, il aurait fait détruire le sanctuaire d'Aphrodite d'Aphaca (sur le territoire de la cité de Byblos) et fermer celui de la colonie romaine d'Héliopolis, les deux lieux étant présentés comme jumeaux dans la dépravation ${ }^{31}$. En Cilicie enfin, il se serait attaqué

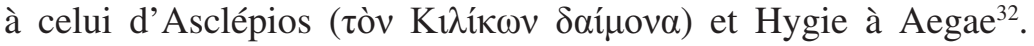
La géographie couverte par la liste (la Palestine, la Phénicie et sa porte cilicienne au nord) est circonscrite au diocèse d'Orient entré dans le pouvoir de Constantin en 324. Elle ne désigne pas de politique d'éradication des lieux de culte et des pratiques païens, qui se lira dans des documents ultérieurs ${ }^{33}$. Assurément le ton de l'édit (nomos) envoyé « aux habitants de la province de Palestine» à l'automne 324 et de la lettre plus générale «aux habitants des provinces orientales »-tous deux reproduits par Eusèbe ${ }^{34}$ - est ferme, mais affiche une tolérance conforme à l'esprit des mesures

29. Doron Bar, «Continuity and Change in the Cultic Topography of Late Antic Palestine », From Temple to Church, p. 275-298, donne un panorama général.

30. Eusèbe de Césarée, Vie de Constantin (dorénavant: VC), III, 25-26 et 51-53 et à sa suite Sozomène, $H E$, II, 1 et 4.

31. Eusèbe de Césarée, VC, III, 55, 2-3 et Triakontaeterikos, VIII, 5-7. Selon Sozomène, $H E, \mathrm{~V}, 10,7$, il aurait fait détruire le sanctuaire d'Héliopolis, bien qu'il n'en dise mot en I, 18.

32. Eusèbe de Césarée, $V C$, III, 56, 1. Le dieu n'est pas nommé, mais les rituels incubatoires et la fonction curative sont décrits ; cf. Eusebius, $V C$ (éd. Cameron - Hall), p. 303, n. 4.

33. Dans la tradition sibylline chrétienne représentée par l'oracle de Baalbek, dont la première version $\left(c\right.$. 378-390) fut développée au début du $\mathrm{VI}^{\mathrm{e}}$ siècle par un chrétien d'Héliopolis, ces défenses et destructions sont étendues à « tous les temples des gentils et les autels du Liban et leurs sacrifices », mais sans allusion à des pratiques immorales, Paul J. Alexander, The Oracle of Baalbek. The Tiburtine Sibyl in Greek Dress, Washington, Dumbarton Oaks ( "Dumbarton Oak Studies », 10), 1967, p. 14, 1. 84-85.

34. Eusèbe de Césarée, $V C$, II, 24-42 et 48-60. 
de 312-313: "que ceux qui s'y [les "saintes lois"] soustraient possèdent, s'ils le veulent, les temples du mensonge $»^{35}$.

À Césarée Maritime, capitale de la province de Syrie-Palestine, le temple d'Auguste construit par Hérode ne semble avoir accueilli l'église octogonale qu'au $\mathrm{VI}^{\mathrm{e}}$ siècle, avec peut-être un bâtiment intermédiaire vers 420, dont la destination religieuse reste à démontrer ${ }^{36}$. Son rôle dans le culte impérial l'a sans doute préservé. La liste eusébienne est concentrée sur quelques sanctuaires exemplaires, propres à cristalliser le message de renversement religieux; d'où les distorsions entre ces textes et les réalités historiques $^{37}$. La motivation missionnaire, ancrée dans la tradition biblique et qui vise donc aussi les Juifs, est transparente pour les deux sanctuaires judéens, Mambré et Jérusalem : l'icône constantinienne dont Eusèbe sculpte le portrait ${ }^{38}$ rend ces lieux à leur vrai Dieu, Jésus-Christ, préfiguré au chêne de Mambré ${ }^{39}$ et mort et ressuscité au Golgotha à Jérusalem. Des raisons similaires rendent compte des trois autres lieux incriminés. Héliopolis et Aphaca étaient des sanctuaires d'Aphrodite; or, Eusèbe de Césarée ne manque jamais de souligner les liens d'Adonis (un «faux» ressuscité) avec la déesse, au point qu'il réserve à cette dernière le Golgotha, alors qu'elle n'était probablement que la «colocataire »

35. Eusèbe de Césarée, VC, II, 56, 2 ; voir aussi 60, 2, et Giorgio Bonamente, «Sulla confisca dei beni mobili dei templi in epoca costantiniana », éd. Giorgio Bonamente, Franca Fusco, Costantino il Grande dall'Antichità all'Umanesimo, t. 1, Macerata, Università degli studi di Macerata, 1992, p. 171.

36. Kenneth G. Holum, «The Temple Platform: Progress Report on the excavations », Caesarea Papers, 2. Herod's temple, the provincial governor's Praetorium and granaries, the later harbor, a gold coin hoard, and other studies, éd. K.G. Holum, Avner Raban, Joseph Patrich, Portsmouth (RI), «Journal of Roman Archaeology, Supplementary Series, 35 », 1999, p. 12-34 ; K. G. Holum, Jennifer A. Stabler, Eduard Gordon Reinhardt, Caesarea Reports and Studies. Excavations 1995-2007 within the old city and the ancient harbor, Oxford, Archaeopress («BAR International Series », 1784), 2008, p. 1-40.

37. Voir Eusèbe de Césarée, $V C$, II, 45, 1, et Martin Wallraff, « Die antipaganen Massnahmen Konstantins in der Darstellung des Euseb von Kaisarea », Spätantike Staat und religiöser Konflikt, p. 7-18.

38. Ulrich Gotter, « Rechtgläubige - Pagane - Häretiker. Tempelzerstörungen in der Kirchengeschichtsschreibung und das Bild der christlichen Kaiser », From Temple to Church, p. 43-59.

39. Voir Marie-Odile Bouldnois, " "Trois hommes et un Seigneur" : lectures trinitaires de la théophanie de Mambré dans l'exégèse et l'iconographie », Studia Patristica, t. 39, éd. Frances M. Young, Mark J. Edwards et Paul Parvis, Louvain, Paris, Dudley, Peeters, 2006, p. 193-201. 
poliade du Capitole de la colonie d'Aelia ${ }^{40}$. Le temple d'Aegae (dont la date de destruction diverge dans les sources du IV ${ }^{\mathrm{e}}$ siècle ${ }^{41}$ ) répond à cette même problématique salutaire : à l'époque tardive, Asclépios fut crédité d'une dimension sotériologique qui déborda sa fonctionnalité thérapeutique et a pu le faire apparaître comme une figure rivale du Christ : « il était au contraire le corrupteur des

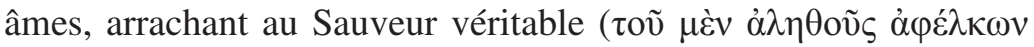
$\left.\sigma \omega \tau \tilde{\eta} \rho \varsigma_{)}\right)$et précipitant dans l'erreur athée ceux qu'il était facile de tromper $»^{42}$.

Bref, ces cinq lieux de culte abritaient des puissances divines considérées comme singeant le vrai message théologique - celui de la résurrection - et patronnant donc des pratiques rituelles réputées infâmes, puisque telle était l'image chrétienne d'Aphrodite et plus généralement des dieux païens ${ }^{43}$. La construction apologétique de la liste, et plus largement des témoignages chrétiens dont nous sommes dépendants, pouvait conduire les auteurs chrétiens à prendre des libertés avec l'identité des occupants païens des temples (comme à Aelia Capitolina), ou avec les pratiques que ces lieux accueillaient, moins dépravées qu'ils ne le décrivent (comme à Héliopolis et Aphaca). Il demeure que le biographe de Constantin appuie son discours théologique en faveur du seul vrai Dieu sur des lieux de culte/espaces paradigmatiques: "In the midst of his Life of Constantine Eusebius has inserted a book of signs, but unlike the signs in the Gospel of John, which were miracles, those in Eusebius' book are places $»^{44}$.

40. Nicole Belayche, Iudaea-Palaestina. The Pagan Cults in Roman Palestine (Second to Fourth Century), Tübingen, Mohr Siebeck (« Religion der Römischen Provinzen », 1), 2001, p. 142-154.

41. Selon Libanios, Pro templis (Oratio 30), 39, la destruction du temple reviendrait à Constance II.

42. Eusèbe de Césarée, VC, III, 56, 1. Voir Pierre Chuvin, Chronique des derniers païens, Paris, Les Belles Lettres, $1991^{2}$, p. 40-41. De même pour Dionysos, voir Wiktor A. Daszewski, Dionysos des Erlöser, Mayence, Zabern, 1985.

43. Procédant de la même idéologie, Jérôme imaginera la grotte de Bethléem souillée par un culte d'Aphrodite, Lettres 58, 3, 5 (Veneris amasius plangebatur). Voir Augustin, Cité de Dieu, VII, 26.

44. Robert L. Wilken, Land called Holy: Palestine in Christian History and Thought, New Haven, Londres, Yale University Press, 1992, p. 90. Voir B. Caseau, « ПO $\Lambda$ EMEIN $\Lambda \mathrm{I} \Theta O I \Sigma$. La désacralisation des espaces et des objets religieux païens durant l'Antiquité tardive », éd. Michel Kaplan, Le sacré et son inscription 


\section{Trois Protagonistes divins à Aelia Capitolina selon Eusèbe}

Le Capitole de la colonie romaine d'Aelia Capitolina, qui a remplacé Jérusalem pour l'administration romaine, est un premier cas intéressant parce qu'il combine une destruction avérée et une argumentation dans laquelle le combat contre les dieux païens couvre un objectif théologique plus fondamental. Il intervient dans un ensemble narratif thématisé au livre III de la biographie/hagiographie eusébienne. L'épisode précède la liste des églises construites par l'empereur (47-51), suivie des trois chapitres (54-56) consacrés aux actions religieuses antipaïennes de Constantin au Levant (récupération d'objets et d'éléments architecturaux dans des temples et démolition des temples d'Aegae et d'Aphaca), et de l'éradication par le chevalier de la nouvelle foi - tel un «aigle volant à travers le ciel $»^{45}$ de pratiques païennes ridicules et vaines (57) et dépravées à Héliopolis (58).

Contrairement à la tradition historiographique qui a majoritairement situé le Capitole sur le mont du Temple, en se fondant sur un passage ambigu de Dion Cassius dans la version abrégée de Xiphilin au $\mathrm{XI}^{\mathrm{e}}{ }^{\text {siècle }}{ }^{46}$ et sur des restes de chapiteaux d'époque romaine retrouvés sur le mont, il se situait plus probablement à l'ouest du cardo maximus, sur le côté nord du forum (l'actuel bazar du Muristan) dont les fondations sont visibles aujourd'hui à l'intérieur de l'église russe, «sur l'emplacement le plus haut » selon les conseils de Vitruve ${ }^{47}$. Là se trouvait la colline du Golgotha, réputée être le lieu de la Passion depuis les années 180, à suivre l'évêque pèlerin Méliton de Sardes («au milieu de la rue

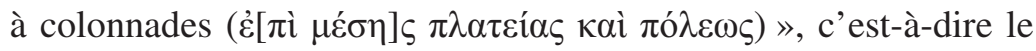

dans l'espace à Byzance et en Occident. Études comparées, Paris, Publications de la Sorbonne, 2001, p. 61-123.

45. Eusèbe de Césarée, $V C$, III, 55, 1.

46. Dion Cassius-Xiphilin, LXIX, 12, 1-2. Les autres sources n'évoquent que des statues d'Hadrien et d'Antonin le Pieux sur le mont du Temple, Eusèbe de Césarée, Démonstration évangélique, VIII, 3, 12 ; Cyrille de Jérusalem, Catéchèse, XVI, 18, 7 (en 347) ; Jérôme, In Sophonias I, 15. Pourtant dès 1912, LouisHugues Vincent et Félix-Marie Abel, Jerusalem. Recherches de topographie, d'archéologie et d'histoire, t. 2: Jerusalem nouvelle, Paris, J. Gabalda, p. 34, proposaient sa localisation sur le Golgotha.

47. Vitruve, Sur l'architecture, I, 7, 1. 
$(a r d o)^{48}$. Seuls les écrits chrétiens évoquent ce bâtiment, et, à la suite d'Eusèbe, sont unanimes à présenter la construction d'un temple à cet emplacement comme la volonté romaine impie d'abolir la mémoire de la passion du Sauveur et de souiller « cette

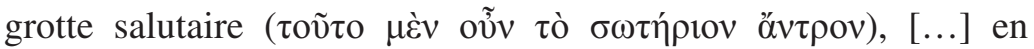
édifiant au démon insatiable d'Aphrodite une ténébreuse caverne

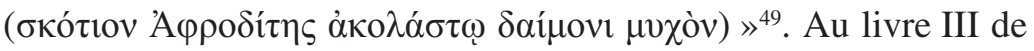
sa Vie de Constantin, Eusèbe relate en quinze chapitres (25-40) la construction de la basilique constantinienne servant d'écrin à l'Anastasis. Il cite la lettre que Constantin envoya, après le concile de Nicée, à Macaire, évêque de Jérusalem, et à tous les gouverneurs de province du diocèse d'Orient, pour ordonner les travaux, donc la destruction du temple, et susciter les dons. L'impact local, voire impérial (car la basilique n'est consacrée qu'en 335), de la destruction fut sans doute peu perceptible alors dans les religions à Aelia Capitolina. L'essentiel de la population païenne de la colonie était constitué des familles de vérérans installés et de la $\mathrm{X}^{\mathrm{e}}$ légion dont le castrum occupait le mont Sion, entouré sans doute de toute une population adventice, comme tout camp légionnaire. Or la légion était partie à Aila/Eilat sur la mer Rouge lors des réformes de Dioclétien, et il est probable que les rituels au temple de Jupiter manquaient désormais d'éclat et d'assistants ${ }^{50}$.

Dans le récit, tout respire le miracle du combat religieux habité par la charis dont l'empereur, par trois fois dépeint comme « inspiré du ciel » ${ }^{51}$, est gratifié par le Sauveur : «ce lieu sacré que,

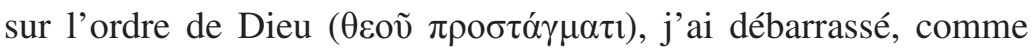

48. Méliton de Sardes, Homélie sur la Pâque, 94 (voir Anthony E. Harvey, «Melito and Jerusalem », Journal of Theological Studies, t. 17, fasc. 2, 1966, p. 401-404, et Joan E. Taylor, Christians and the Holy Places. The Myth of Jewish-Christian Origins, Oxford, Clarendon Press, 1993, p. 117-122). Pour la démonstration détaillée, N. Belayche, Pagan Cults, p. 142-149.

49. Eusèbe de Césarée, $V C$, III 26, 2 et 3 (trad. Marie-Josèphe Rondeau, Paris, Le Cerf, «Sources chrétiennes », 559, 2013). Voir Théodoret de Cyr, HE, I, 16, 5. Frank R. Trombley, Hellenic Religion and Christianization, t. 1, Leyde, New York, Brill (Religions in the Graeco-Roman World, 115), 1995, p. 112, prenant Eusèbe au pied de la lettre, parle sans preuve de « cave-temple».

50. Les ouvrages édités par J. Hahn, S. Emmel, U. Gotter et L. Lavan, M. Mulryan, cités supra n. 4, ont insisté sur les désaffections de temples dès le $\mathrm{III}^{\mathrm{e}}$ siècle pour des raisons conjoncturelles et non religieuses.

51. Eusèbe de Césarée, $V C, 26,6 ; 27,1 ; 30,4$. 
d'un fardeau placé sur lui, de l'ignoble idole qu'on lui avait ajoutée $»^{52}$. La monumentalité du sanctuaire capitolin ne peut que se reconstruire en creux à partir des récits de sa destruction. Au II $^{\mathrm{e}}$ siècle, la colline fut nivelée de façon à créer une esplanade qui accueillerait un temple de type gréco-romain ${ }^{53}$. Chez Eusèbe, qui put voir les travaux de construction, «les ouvrages machinés par la tromperie furent abattus de fond en comble ${ }^{54}$. Sozomène, qui poursuivit la première Histoire ecclésiastique, file cette tradition. « Ni ce bois [de la croix] ni le saint tombeau n'étaient aisés à découvrir. Car les païens [...] avaient comblé sous une masse de terre (ímò $\pi \mathrm{o} \lambda \lambda \tilde{\omega} \chi \omega \dot{\omega} \mu \alpha \tau)$ le lieu de la tombe et ils l'avaient

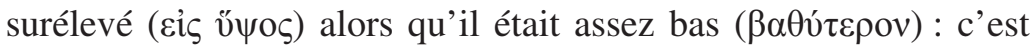
ainsi qu'il se voit encore aujourd'hui ».

L'historien $\mathrm{du} \mathrm{V}^{\mathrm{e}}$ siècle ajoute une précision sur le péribole du téménos, qui devait accueillir les deux temples de la triade capitoline et d'une Tychè-Aphrodite-Artémis ${ }^{55}$. «Ils avaient entouré d'une enceinte tout l'emplacement de la Résurrection et du

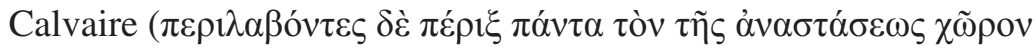

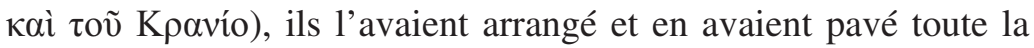
surface $»^{56}$.

Les terrassements destinés à aménager la plateforme du temple consistèrent donc en des travaux de comblement et de surélévation, et non d'éradication des structures antérieures - dont la tombe de Jésus (inconnue alors des chrétiens si Méliton est un terminus post quem), d'où la possibilité de l'invention miraculeuse lors des travaux de déblaiement. Chaque épisode est marqué au sceau de la providence divine et tout le récit des travaux est placé sous le sceau de la vanité de ces ténèbres démoniaques à chercher à occulter la lumière de la vraie Vie. Le lieu aurait été découvert «contre toute espérance $\left.\left(\pi \alpha \rho^{\prime} \dot{\varepsilon} \lambda \pi \hat{i} \delta \alpha \pi \tilde{\alpha} \sigma \alpha \nu\right) »\right)$, et sa mise au

52. Ibid., III, 30, 4 ; voir aussi 25.

53. Voir Magen Broshi et Gabrial Barkay, «Excavations in the Chapel of St Vartan in the Holy Sepulchre », Israel Exploration Journal, t. 35, 1985, p. 108-124 ; Shimon Gibson et Joan E. Taylor, Beneath the Church of the Holy Sepulchre Jerusalem, Londres, Palestine Exploration Fund («Palestine Exploration Fund monograph », 1), 1994, p. 65-71 (je me sépare de leurs conclusions qui situent le Capitole sur l'ancienne forteresse Antonia, «the perfect location », p. 70, à l'issue d'un raisonnement insuffisamment critique envers les sources littéraires).

54. Eusèbe de Césarée, VC, III, 26, 7 et 27, 1.

55. Voir N. Belayche, Pagan Cults, p. 144-153.

56. Sozomène, $H E$, II, 1,3 . 
jour est saluée comme une mimèsis de la résurrection, voire sa revification :

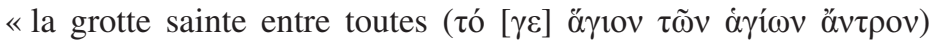
recouvra son caractère d'image fidèle du retour du Sauveur à la vie

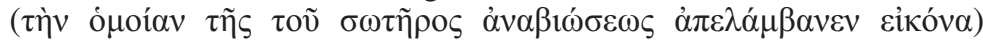
- c'est pourquoi après la plongée dans les ténèbres elle émergeait à

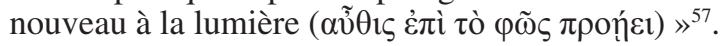

Un écho épigraphique rare (cité en exergue), gravé au-dessus de la porte de l'église Saint-Georges à Zorava en Trachonitide au début du $\mathrm{VI}^{\mathrm{e}}$ siècle, atteste que l'expression chrétienne de cette 《révolution constantinienne ${ }^{58}$ s'est fixée dans ce même lexique et dans ce même imaginaire spatial. Dans la Vie eusébienne, la construction de la basilique du Saint-Sépulcre est un morceau de bravoure destiné à médiatiser la rupture religieuse de la politique de l'empereur, fondatrice d'un nouus saeculorum ordo ${ }^{59}$. Mais le théologien l'illustre d'autant plus que c'est le joyau de la courte liste des sanctuaires détruits, et que le lieu n'implique pas seulement les païens, mais aussi et d'abord les Juifs. Le récit eusébien rapporte l'événement comme l'avènement d'un nouveau temps, un renversement total avec destruction violente à fins de purification. Les matériaux devaient être jetés au loin pour

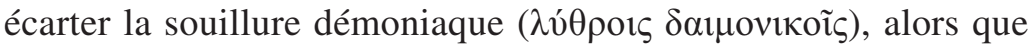
l'archéologie démontre le remploi d'un certain nombre de blocs ${ }^{60}$. Mais sous l'écume païenne qui dramatise le récit, le principal enjeu semble ailleurs, pour qui lit Eusèbe de près. Le lieu comme la construction gagnaient une valeur symbolique unique du fait qu'ils manifestaient «cette toute nouvelle Jérusalem annoncée par les oracles des Prophètes » face à l'ancienne ( $\dot{\alpha} v \tau \imath \pi \rho o ́ \sigma \omega \pi \mathrm{s}$

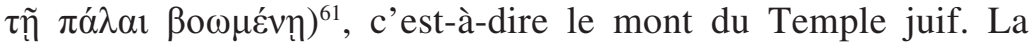
rhétorique récurrente qui oppose l'avant ( $\pi \rho \operatorname{có}_{\varepsilon \rho \rho \mathrm{v})}$ ) au présent

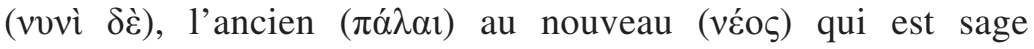
$(\sigma \omega ́ \varphi \rho \omega v)$, renvoie à une double temporalité, appuyée sur une

57. Eusèbe de Césarée, $V C$, III, 28.

58. Voir Raymond Van Dam, The Roman Revolution of Constantine, New York, Cambridge, Cambridge University Press, 2007, p. 283-352.

59. Ammien Marcellin, XXI, 10, 8.

60. Les remblais nord du Saint-Sépulcre sont en partie constitués par les matériaux des constructions détruites pour ériger le complexe constantinien : voir Corpus Inscriptionum Iudaeae/Palaestinae, I, 2, nº 716.

61. Eusèbe de Césarée, $V C$, III, 33, 1-2. 
conception théologique ${ }^{62}$ - et accessoirement sur un fond de rivalités entre les sièges épiscopaux de Jérusalem et de Césarée Maritime $^{63}$. Les féroces accents contre les idoles et leurs repaires s'adressent à une temporalité courte, anecdotique au regard du plan divin. L'évêque situe fondamentalement la nouvelle ère dans la perspective théologique de l'accomplissement que Jésus devenu Christ a opéré, remplaçant le lieu de l'ancienne Loi par celui de «la bonne nouvelle de la régénération révélée par le

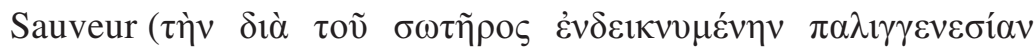

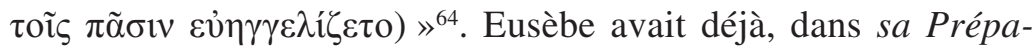
ration évangélique, situé le christianisme et son exception comme étant ce troisième terme : «ce qu'on pourrait appeler proprement le christianisme, qui n'est ni l'hellénisme ni le judaïsme, mais une nouvelle et véridique science divine qui, par sa dénomination même, met en avant sa nouveauté (tèn kainotomian) $»^{65}$.

Ce cas paradigmatique d'une fin programmée de temple nous instruit moins sur la «fin des cultes » qu'il n'exemplifie la stratégie d'un discours politico-théologique visant à illustrer la mutation, présentée comme radicale, que pouvait représenter le «premier empereur chrétien », précisément parce que la politique suivie ne fut pas conflictuelle. Depuis le changement de paradigme qu'ont initié les études de Peter Brown principalement, les enquêtes de terrain montrent que les destructions violentes furent souvent liées à des configurations locales particulières : à côté du Capitole d'Aelia Capitolina, l'exemple le mieux attesté est celui du Sarapieion d'Alexandrie à la fin du IV ${ }^{\mathrm{e}}$ siècle, qui a suscité bien davantage une mise en rituel et en discours de la perception d'un

62. Pour l'accent sur la résurrection plutôt que sur la passion du crucifié (« the deviousness of Eusebius »), voir Stephan Borgehammar, How the Holy Cross was Found. From Event to Medieval Legend with an Appendix of Texts, Stockholm, Almqvist and Wiksell («Bibliotheca Theologiae Practicae », 47), 1991, p. 105-108 et p. 115 suiv.

63. Voir le canon 7 du concile de Nicée : I Canoni dei concili della Chiesa antica, t. 1 : Concili greci, dir. Angelo di Berardino, Rome, Institutum Patristicum Augustinianum («Studia ephemeridis Augustinianum», 95), 2006, p. 23. Plus largement, Edward D. Hunt, «Constantine and Jerusalem», Journal of Ecclesiastical History, t. 48, 1997, p. 405-424.

64. Eusèbe de Césarée, $V C$, III, 33, 2.

65. Eusèbe de Césarée, La Préparation évangélique, I, 5, 12, trad. É. des Places et J . Sirinelli (« Sources Chrétiennes », 206). 
dieu païen ${ }^{66}$, qui est inexistante dans le récit à Aelia où l'enjeu de fond n'était plus celui du paganisme malgré l'apparence du récit.

\section{Aphaca/Héliopolis : \\ LA CONSTRUCTION D'UN MYTHE HISTORIOGRAPHIQUE}

Le deuxième cas d'étude d'époque constantinienne concerne les deux temples d'Aphrodite à Aphaca et Héliopolis en SyriePhénicie. À nouveau, seule la tradition chrétienne, et Eusèbe le premier, nous informe sur la destruction de l'un et la fermeture de l'autre. Les deux événements doivent être lus en parallèle, du fait de leur proximité géographique ${ }^{67}$, parce que les historiens ecclésiastiques eux-mêmes, Socrate et Sozomène, ont établi le $\operatorname{lien}^{68}$, et parce que les récits développent une trame dramatique commune : des pratiques licencieuses et dépravées.

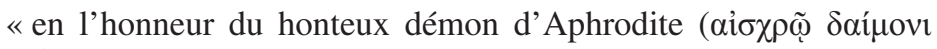
A $\varphi \rho o \delta i ́ r \eta \zeta)$, dans une région montagneuse du Liban, à Aphaca. C'était

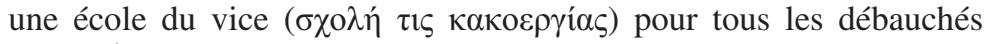

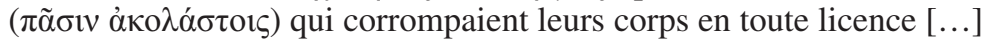
Il [Constantin] jugea qu'un tel temple était indigne de la lumière du soleil et ordonna qu'on le fit disparaitre jusqu'aux fondations avec les

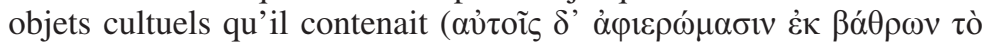
$\pi \tilde{\alpha} \nu \grave{\alpha} \varphi \alpha v 1 \sigma \theta \tilde{\eta} v \alpha \iota \kappa \varepsilon \lambda \varepsilon \varepsilon v \varepsilon l){ }^{69}$.

« À Héliopolis de Phénicie [Baalbek dans l'Anti-Liban] où ceux qui honoraient, sous le nom d'Aphrodite, les plaisirs de la débauche permettaient auparavant à leurs femmes et à leurs filles de se prostituer librement. Désormais, édictée par l'empereur ( $\pi \alpha \rho \grave{\alpha} \beta \alpha \sigma \iota \lambda \varepsilon ́ \omega \varsigma)$, une nouvelle loi relative à la tempérance interdisait l'effronterie des anciennes coutumes $»^{70}$.

66. Johannes Hahn, « The Conversion of the Cult Statues: the Destruction of the Serapeum 392 A.D. and the Transformation of Alexandria into the "ChristLoving City" », From Temple to Church, p. 335-365.

67. Zosime, Histoire nouvelle, I, 58, 1, situe Aphaca «à mi-chemin entre Héliopolis et Byblos ».

68. Socrate, $H E, \mathrm{I}, 18,10$ : «D'une manière identique à Aphaca du Liban »; Sozomène, $H E, \mathrm{I}, 8,6$.

69. Eusèbe de Césarée, VC, III, 55, 2-4 (=Louanges de Constantin / Triakontaéterikos, VII, 5-7).

70. Ibid., III, 58, 1-2. Selon Sozomène, HE, V, 10, 7, il y eut aussi destruction :

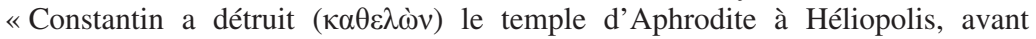
de construire dans cette ville une église, et interdit par une loi de pratiquer la prostitution coutumière ». 
L'accusation de prostitution sacrée qui déclenche, selon l'évêque, la décision de Constantin, contribue à obscurcir le dossier, car la prostitution sacrée dans l'Antiquité, a fortiori à époque si tardive, est elle-même mal, pour ne pas dire pas, attestée ${ }^{71}$ et relève souvent du mythe historiographique. Depuis l'article séminal de Daniel Arnaud en 1973, plusieurs travaux l'ont confirmé, dont récemment encore ceux de Vinciane Pirenne Delforge ${ }^{72}$. Pour Aphaca, outre les auteurs chrétiens, Ps.?-Lucien dans son traité Sur la déesse syrienne, avait déjà signalé lors des Adonies des pratiques de prostitution "profane» avec des hommes étrangers

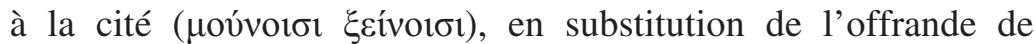
leur chevelure (sacrifice connu d'action de grâce ou de substitution, par exemple pour ceux qui ont été sauvés d'un naufrage), dont «la rémunération devient un sacrifice à Aphrodite

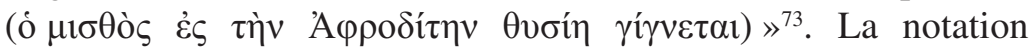
relative aux étrangers est remarquable pour ce qu'elle révèle du contrôle social qui pesait sur ces relations extra-ordinaires ${ }^{74}$. Elle a pu être inspirée au classiciste Lucien par Hérodote, décrivant des coutumes babyloniennes dans un temple de Myllitta/ Aphrodite : «toute femme du pays doit, une fois en sa vie (ö $\pi \alpha \xi$

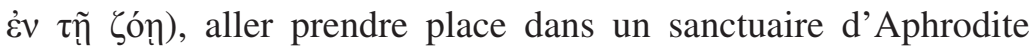

71. Athanase d'Alexandrie, Contre les païens, 26, signale vaguement cette

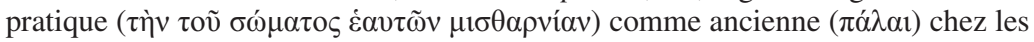
Phéniciens ; voir aussi Augustin, Cité de Dieu, IV, 10.

72. Daniel Arnaud, «La prostitution sacrée en Mésopotamie, un mythe historiographique ? ", Revue de l'Histoire des Religions, t. 92, 1973, p. 111-115, et Marie Beard et John Henderson, "With This Body I Thee Worship: Sacred Prostitution in Antiquity », Gender and the Body in Mediterranean Antiquity, éd. Maria Wyke, Oxford, Clarendon Press, 1997, p. 480-503. Pour la difficile distinction entre prostitution sacrée et profane, voir Vinciane Pirenne-Delforge, L'Aphrodite grecque. Contribution à l'étude de ses cultes et de sa personnalité dans le panthéon archaïque et classique, Liège, Centre international d'étude de la religion grecque antique («Supplément de Kernos », 4), 1994, p. 116-121, et son compte rendu de Stephanie Budin, The Myth of Sacred Prostitution in Antiquity, New York, 2008, en ligne (http://bmcr.brynmawr.edu/2009/2009-04-28.html).

73. Ps.?-Lucien, Sur la déesse syrienne, 8, trad. Brigitte Soyez, Byblos et la fête des Adonies, Leyde, Brill («Études Préliminaires aux religions orientales dans l'Empire romain », 60), 1977, p. 13-14.

74. En Lydie (Hérodote, Histoires, I, 93); à Chypre (Jean Rudhardt, "Quelques notes sur les cultes chypriotes, en particulier sur celui d'Aphrodite », cité par V. Pirenne-Delforge, Aphrodite, p. 118-119), la prostitution des jeunes filles permettait de rassembler la dot de la future mariée. Pour l'offrande de la virginité, Walter Burkert, Homo necans, Berkeley, University of California Press, 1983, p. 62-63. 


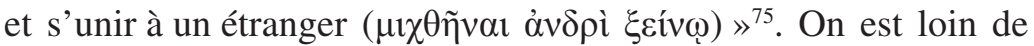

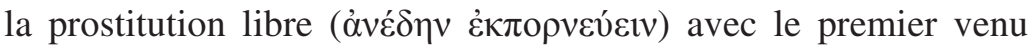

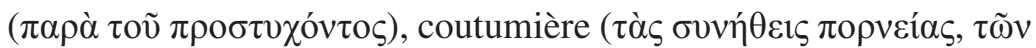

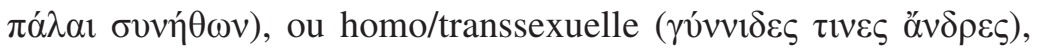
dépeinte par les auteurs chrétiens pour les deux sanctuaires phéniciens ${ }^{76}$. Les unions débridées qu'ils dénoncent renvoient en revanche à un topos récurrent de l'idolâtrie, théologiquement réputée pour se combiner avec l'impudicité et des conduites dépravées ${ }^{77}$.

L'examen comparé de ces deux «fins de cultes», dont les descriptions des pratiques rituelles se superposent ${ }^{78}$, conduit à conclure que la décision de fermeture que Constantin aurait prise pour Héliopolis/Baalbek est en réalité un doublet littéraire, créé par Eusèbe et transmis par sa postérité littéraire, des mesures prises contre Aphaca - dont «l'école du vice » est tout sauf démontrée ${ }^{79}$. De fait le tableau ignominieux qui est prêté à la population héliopolitaine recoupe mal la documentation historique et littéraire sur cette colonie romaine ${ }^{80}$, qui disqualifie l'assertion de Socrate relative à une structure sociale établie

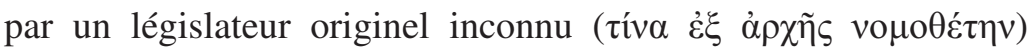

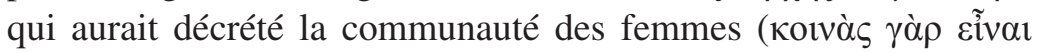

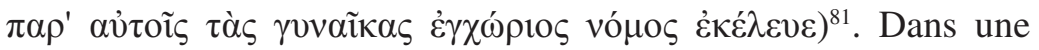
colonie de droit romain où le statut des personnes a des implications sociopolitiques et fiscales, cette communauté des femmes paraît bien imaginative. Les prêtres de Jupiter héliopolitain étaient des notables romains dont Macrobe, dans la première moitié du $\mathrm{V}^{\mathrm{e}}$ siècle, souligne la pureté après une longue continence (longi

75. Hérodote, Histoires, I, 199.

76. Eusèbe de Césarée, $V C$, III, 58, 1-2 et 55, 3 ; Sozomène, $H E, \mathrm{~V}, 10,7$ et I, 8,6 .

77. Par exemple Paul, 1 Corinthiens, 6, 9-11 et 1 Thessaloniciens, 4, 3-4.

78. Pour l'étude détaillée de ce dossier, N. Belayche, «Constantin a-t-il légiféré contre des pratiques rituelles à Héliopolis (Baalbek)?», Dieu(x) et Hommes. Mélanges en l'honneur de Françoise Thélamon, éd. Sylvie Crogiez-Pétrequin, Rouen, Publications des Universités de Rouen et du Havre, 2005, p. 101-112.

79. Luce Piétri, Eusèbe de Césarée, Vie de Constantin ( Sources chrétiennes », 559), p. 430-431, n. 1, crédite mon analyse.

80. Pour l'étude la plus récente sur le culte de Vénus à Héliopolis, Anne-Rose Hošek, Territoires et religions en contacts : la colonie romaine de Berytus, de sa fondation au III siècle de notre ère, Thèse, École Pratique des Hautes Études, 2012 (publiée sur HAL : https://hal.archives-ouvertes.fr/tel-01552363v1).

81. Socrate, $H E, \mathrm{I}, 18,7$. 
temporis castimonia puri) avant d'approcher le dieu ${ }^{82}$. Sa collègue féminine, Vénus héliopolitaine - Aphrodite sur une seule inscription ${ }^{83}$-, était une divinité romaine du fait de son statut dans la cité, et n'était pas servie par des hiérodules prostituées d'après la documentation dont nous disposons ${ }^{84}$. Les dédicaces familiales (pro salute du mari, de sa femme et de leurs enfants) qui proviennent du sanctuaire ${ }^{85}$ ne laissent rien soupçonner d'une possible incertitude sur l'identité des parents telle que Socrate l'énonce ${ }^{86}$.

Ce doublet Aphaca/Héliopolis ne fait que conforter l'existence de stratégies narratives systématiques - diabolisation et éradication - destinées à illustrer la figure du «premier empereur chrétien » plus qu'à attester de la fin des cultes, dès qu'il est question de sanctuaires païens pendant le règne de Constantin.

\section{ILluSTRER Un ÉvêQUe AU MARneion de GAZA}

Le dernier cas choisi fait un saut chronologique et conduit à l'achèvement de la construction chrétienne d'un discours sur la «fin des cultes» à partir de l'argument des sanctuaires. Il appartient à l'empire officiellement chrétien depuis 380-392. Des donnes fondamentales ont changé : d'après leurs hagiographies, les saints évêques soutiennent désormais les empereurs dans la lutte contre les démons ${ }^{87}$, quand ils ne s'impatientent pas de l'attentisme des autorités politiques. Du moins c'est ce qui ressort

82. Macrobe, Saturnales, I, 23, 13.

83. Youssef Hajjar, La triade d'Héliopolis-Baalbeck. Son culte et sa diffusion à travers les textes littéraires et les documents iconographiques et épigraphiques, t. 1, Leyde, Brill («Études préliminaires aux religions orientales dans l'empire romain », 59), 1977, $\mathrm{n}^{\circ} 78$.

84. Elles sont connues dans d'autres sanctuaires, par exemple à Corinthe, Strabon, VIII, 6, 21 et XII, 3, 36, sanctuaire «fréquenté par les marchands et les soldats, donc des étrangers aussi ; voir Pierre Debord, «L'esclavage sacré : état de la question ", Actes du Colloque 1971 sur l'esclavage, Paris, Besançon, Annales littéraires de l'Université de Besançon («Centre de recherches d'histoire ancienne », 6), 1972, p. 135-150 (p. 140 pour la prostitution sacrée).

85. Y. Hajjar, La triade d'Héliopolis-Baalbeck, $\mathrm{n}^{\circ} 20-22$; cf. aussi no ${ }^{\circ} 64-65$.

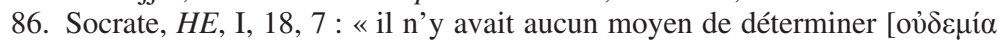

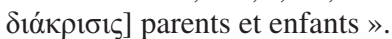

87. Voir Bernadette Cabouret, « Application de la loi et initiatives individuelles : le témoignage de Libanios d'Antioche », Chrétiens persécuteurs, p. 189-219. 
de la Vie de Porphyre évêque de Gaza réputée écrite par Marc le Diacre $^{88}$, qui met en scène un empereur, Arcadius, et un évêque Porphyre. Nombre de savants ont déjà signalé que l'œuvre était largement fictive ${ }^{89}$. Mais ce qui est précisément important pour la présente étude est que les récits de destruction élaborés par Eusèbe (et à partir de lui) au sujet de sanctuaires attestés ont abouti à construire un modèle de "fin des cultes », dans lequel désormais le rôle de «chevalier de la foi » est assumé par l'évêque et plus par l'empereur. Le Marneion - le sanctuaire du theos patrios Marnas, un «seigneur de la pluie» atmosphérique interprété en Zeus comme tous les grands dieux locaux du Proche-Orient, qui incarnait l'identité de la cité comme tant de grands temples civiques ${ }^{90}-$ est un sanctuaire attesté, mais pas sur le terrain. Selon l'hagiographe, sa destruction serait intervenue dans la première décennie $d u v^{e}$ siècle et aurait nécessité le recours à l'intervention impériale, tiède pendant longtemps ${ }^{91}$, et à la coercition par des troupes envoyées de Constantinople et de Césarée.

Lors de la dernière attaque de Porphyre en 402 (selon les efforts de reconstruction), le débat qui parcourut les chrétiens, tout fictif soit-il (il est tranché par un signe de Dieu), est révélateur de nombreuses situations locales dans lesquelles le temple servait d'emblème local. «Les uns proposaient de le détruire de fond en comble, les autres de le brûler entièrement et d'autres encore de purifier l'endroit et de le consacrer pour en faire une église de Dieu $\gg^{92}$.

88. Sur les problèmes posés par ce texte, voir depuis l'édition et traduction d'Henri Grégoire et Marc-Antoine Kugener dans la Collection des Universités de France en 1930, La conversion de Gaza au christianisme. La Vie de S. Porphyre de Gaza par Marc le Diacre, éd. Anna Lampadaridi, Bruxelles, Société des Bollandistes ( Subsidia Hagiographica», 95), 2016 (édition choisie pour les références). Elle date p. 19 le texte de 445-556 et considère p. 23 Porphyre comme «un évêque probablement historique ». Timothy D. Barnes, Early Christian Hagiography and Roman History, Tübingen, Mohr Siebeck, 2010, p. 260-283, doute de l'historicité de Porphyre. Selon Aude Busine, «From Stones to Myth. Temple Destruction and Civic Identities in the Late Antique Roman East », Journal of Late Antiquity, t. 6, 2014, p. 325-346, c'est une "fictitious hagiography » (p. 331-332) destinée à construire «a foundation myth for the great church of Gaza » (p. 338 ; moins hypercritique p. 340).

89. Voir note précédente et tout récemment encore le compte rendu de l'ouvrage cité d'Anna Lampadaridi par Sylvain Destephen dans la Revue de l'histoire des religions 234/3, 2017, p. 536-539.

90. CTh XVI, 10, 8, au duc d'Osrhoène.

91. Vita Porphyrii (dorénavant: V. Porph.) 27, 11-15.

92. V. Porph., 66, 2-4, trad. A. Lampadaridi, p. 145. 
Les mentions répétées de Marc le Diacre sur les embûches que Porphyre dut surmonter appartiennent à la stratégie narrative propre à dessiner le portrait de foi et de sainteté de son champion. Pourtant la contextualisation historique invite à reconnaître des matériaux de vraisemblance dans la multitude de notations triviales et quotidiennes, que l'histoire compliquée du texte ${ }^{93}$ et la narration hagiographique ont utilisée pour donner une couleur de véracité à la mise en discours miraculeuse.

Sans détailler le «récit» de l'âpre lutte d'influence entre l'évêque Porphyre et la puissante élite civique païenne, qui réussit à bloquer l'application des lois impériales confiée aux fonctionnaires locaux ${ }^{94}$, on notera que, comme quelques années plus tôt à Apamée de Syrie en 386, l'Église locale, peu nombreuse malgré les conversions ${ }^{95}$, était impuissante sans le bras armé de l'État ${ }^{96}$. Porphyre dut faire appel par deux fois (?) à l'autorité impériale et à sa force de coercition ${ }^{97}$. Plusieurs épisodes narratifs, confirmés pour Alexandrie aussi, rapportent la subornation d'officiers impériaux ${ }^{98}$ qui savaient que l'empereur avançait sans dogmatisme sur le terrain de la lutte contre le paganisme et fermait les yeux sur une application laxiste (sans «hyperbolè») des édits prohibant les sacrifices ${ }^{99}$. De fait, la loi de Théodose II du 14 novembre 435 prescrit encore la transformation chrétienne

93. Pour un « plagiat » de l'HE de Théodoret de Cyr dans le prologue, l'édition d'Henri Grégoire et Marc-Antoine Kugener, Marc le Diacre. Vie de Porphyre, évêque de Gaza, Paris, Les Belles Lettres, 1930, p. XXXIII-XXXVIII.

94. Ce qui est confirmé par $C T h, X V I, 10,12,4$. Après la destruction du temple même, une nouvelle émeute des «païens », contemporaine du regain général de combativité avec l'usurpation d'Attale et de l'édit de tolérance d'Honorius en 409, V. Porph., 95 et 99 ; voir l'édition de Grégoire et Kugener, p. LXVIII-LXX.

95. Porphyre essuie des reproches pour avoir construit une église «bien grande, tandis que les chrétiens de la ville étaient peu nombreux », V. Porph, 93, 2-3.

96. Voir Théodoret de Cyr, HE, V, 21, 5-15, et Sozomène, HE, VII, 15, 12-13 ; Garth Fowden, « Bishops and Temples in the Eastern Roman Empire A.D. 320-435 », Journal of Theological Studies, t. 29, fasc. 1 (n.s.), 1978, p. 53-78 (ici p. 64-66) ; F. R. Trombley, Hellenic Religion, p. 123-129; Aude Busine, «From Stones to Myth », p. 325-346.

97. Ramsay McMullen, Christianizing the Roman Empire, A.D. 100-400, New Haven, Londres, Yale University Press, 1984, p. 86-101.

98. Outre supra n. 94, V. Porph., 21, 19-21. Sur le manque d'empressement de certains officiers, pour Alexandrie Sozomène, HE, VII, 15, 2-5.

99. Voir Emanuele Testa, «Legislazione contro il paganesimo e cristianizzazione dei temple (sec. IV-VI) », Liber Annuus, t. 41, 1991, p. 311-313. 
de «tous les sanctuaires, lieux de culte, temples, s'il en reste encore d'intacts (fana, delubra, templa qua etiam nunc restant integra) ${ }^{100}$; il devait donc en rester. Selon la Vie de Porphyre, l'argument fiscal n'était pas étranger à la politique impériale, ce qui n'a rien d'implausible: Arcadius était soucieux de protéger les intérêts du fisc et des rentrées régulières qui alimentaient plus sûrement le trésor que la confiscation des biens encourue par toute personne reconnue coupable de pratiques sacrificielles et divinatoires ${ }^{101}$. Or Gaza et son port remarquablement bien situé devaient effectivement verser des sommes substantielles ${ }^{102}$. Porphyre aurait donc préparé plus efficacement l'ultime attaque, s'assurant des soutiens de l'évêque métropolitain de Césarée, de l'impératrice Eudoxie en personne, et de la force civile et militaire ${ }^{103}$.

La représentation de la destruction du Marneion de Gaza, mise en regard avec celle du Capitole d'Aelia Capitolina, confirme le poids des rapports de force locaux même face à une politique impériale. De l'Osrhoène à Rome, en passant par Carthage, les lois et rescrits conservés dans le Code Théodosien indiquent que l'application des décisions impériales générales d'interdiction des pratiques païennes et de fermeture ou destruction de temples a varié selon les configurations sociales et religieuses locales, surtout lorsque le temple représentait un référent identitaire. C'est pourquoi la symbolique des espaces cultuels - en l'occurrence la destruction des temples - a pris une telle place dans des discours idéologiques dans lesquels détruire le temple signifie terrasser le démon: « Marnas a été vaincu par le Christ (Marnas uictus est a Christo)» comme l'avait été à Alexandrie Sarapis, «l'auteur de l'erreur (ad subuertendum erroris auctorem) ${ }^{104}$. Dans tout l'empire, la fin des cultes publics fut un phénomène sociopolitique et non

100. CTh., Xvi, 10, 25 .

101. CTh., Xvi, 16, 10, 12, 2 (8 novembre 392).

102. V. Porph., 41, 4-11; voir l'édition de Grégoire et Kugener, p. LXIII.

103. V. Porph., 50, 11-15 et 63, 1-3.

104. Jérôme, Vie d'Hilarion, 11, 11 ; Rufin d'Aquilée, $H E$, II, 23 ; voir aussi à Aegae, Eusèbe de Césarée, $V C$, III, 56, 2. Voir Francesco Massa, « Devotees of Serapis and Christ? A Literary Representation of Religious Cohabitations in the 4th Century », Beyond Conflicts. Cultural and Religious Cohabitations in Alexandria and in Egypt between the 1st and the 6th century, L. Arcari (éd.), Tübingen, Mohr Siebeck, 2017, p. 213-229. 
religieux ${ }^{105}$. C'est avant tout l'attitude des élites locales, et leurs appréciations, divergentes selon les contextes, de leurs intérêts pour la préservation de leur statut, qui les a déterminées à conserver leurs traditions religieuses ou à adhérer à une nouvelle, et qui rend donc compte des phénomènes de conversion. Dans des sociétés civiques qui étaient constituées de multiples réseaux, les élites entraînaient leurs cercles, bien plus sûrement que les évolutions religieuses de l'époque impériale analysées à l'aune de l'individualité.

\section{Conclusion}

Quoi qu'il en soit des acclamations de victoire lors de la mise à bas des temples dans les histoires ecclésiastiques comme dans les récits hagiographiques, la destruction d'un sanctuaire ne signifiait pas ipso facto la « fin des cultes $»^{106}$, sauf lorsque les dévots avaient quitté la ville, comme les soldats à Aelia Capitolina. À la fin du $\mathrm{IV}^{\mathrm{e}}$ siècle, le paganisme restait solidement implanté dans la région gazéenne ${ }^{107}$. À Mambré près de Hébron, les fêtes d'été célébrées autour de l'arbre et du puits sacré d'Abraham sont exemplaires puisque le sanctuaire fait partie de ceux dont Constantin avait ordonné la destruction ${ }^{108}$. «Des idoles [...] sont établies auprès du chêne, un autel se dresse à côté et on y offre constamment des sacrifices impurs », écrit Constantin à Macaire et aux évêques de Palestine pour justifier sa décision ${ }^{109}$. Jérôme et surtout Sozomène, donc au $\mathrm{V}^{\mathrm{e}}$ siècle ${ }^{110}$, en décrivent les rites, sacrifiels pour les païens ${ }^{111}$, mais qui réunissaient aussi juifs et chrétiens «priant le

105. Christophe J. Goddard, Les piétés païennes de l'Italie tardo-antique, thèse dactylographiée, Paris X Nanterre, 2003.

106. Voir Robert A. Markus, The End of Ancient Christianity, Cambridge, Cambridge University Press, 1990, p. 107-109 pour Gaza.

107. Le biographe rapporte les vexations subies par Porphyre à son arrivée, V. Porph., 17, 4-9.

108. N. Belayche, Pagan Cults, p. 96-97. Pour les fouilles allemandes : Evariste Mader, Mambre, die Ergebnisse der Ausgrabungen im heiligen Bezirk Râmet el-Halîl in Südpalästina, 1926-1928, Fribourg, E. Wewel, 1957, p. 288-304 ; voir de même les fouilles en cours de la Mission archéologique française de Mambré (dir. Vincent Michel) avec le Département des Antiquités de Palestine Sud.

109. Eusèbe de Césarée, VC, III, 53, 1.

110. Jérôme, Commentarii in Jeremiam, 31, et Sozomène, HE, II, 4. Voir Evariste Mader, Mambre, p. 288-289.

111. Ibid., p. 137, n ${ }^{\circ} 6$ et pl. LXXVI. 
Dieu de l'univers $»^{112}$, lors d'une panégyrie qui coïncidait avec un marché que le Talmud interdit aux Juifs ${ }^{113}$. La christianisation du lieu, avec construction d'une basilique qui s'appuya sur les murs hadrianéens, n'a donc pas empêché la poursuite des dévotions païennes, peut-être parce que le sanctuaire était à ciel ouvert et sans structure monumentale.

La fonction des discours sur la destruction des temples dans la littérature chrétienne pour signifier le retournement religieux répond à trois objectifs : démontrer la vanité des dieux qui les occupent, dessiner la christianisation comme un changement d'ère, écrite sur une tabula rasa purifiée, et poser leurs promoteurs présumés en saints champions, qu'ils soient empereur ou clerc. En l'absence de possibilité d'enquête ethnographique de terrain, la question de la fin des cultes dans les systèmes ritualistes est toujours délicate, étant donné la nature même des rites, des actes non pérennes. Au IV siècle, la difficulté est décuplée du fait d'une documentation chrétienne à visée polémique et apologétique, qui contribue à brouiller l'information. L'utilisation de modèles interprétatifs construits sur des présupposés plus ou moins objectifs peine à s'affranchir de grandes reconstructions historiques bâties sur des siècles d'historiographie christianocentrée. Sur ce dossier limité à quelques destructions sur ordre impérial, les affichages de communication, qui ont leur propre logique, se heurtent plusieurs fois aux quelques realia qu'on peut glaner. D'autre part les politiques volontaristes en matière religieuse touchent plus facilement la visibilité cultuelle que le quotidien des pratiques. Un dieu meurt lorsqu'il n'a plus de dévot et non lorsqu'on détruit son écrin architectural.

"Contre un dieu, quand on est un homme, vouloir lutter? Quelle audace!» (Euripide, Bacchantes 616-617).

nicole.belayche@ephe.sorbonne.fr

112. Sozomène, HE, II, 4, 3. A. Kofsky, «Mamre : a Case of a Regional Cult ? », Sharing the Sacred, p. 19-30.

113. En effet Hadrien y avait vendu les esclaves juifs de la deuxième révolte, Talmud de Jérusalem, Avodah Zarah 1, 4, 38d (Schwab XI, p. 185) ; Jérôme, Comm. in Jer., 68, 6 et in Zach., 3, 11, 4. 